\title{
Trabajo comunitario y conformación de liderazgos de mujeres indígenas en Valle de San Quintín, Baja California
}

\section{Community work and conformation of leadership of indigenous women in Valle de San Quintín, Baja California}

María de Lourdes Camarena Ojinaga ${ }^{1}$, Concepción Martínez Valdés ${ }^{2}$, Evarista Arellano García $^{3}$ y Christine von Glascoe ${ }^{4}$

\begin{abstract}
Resumen
Este trabajo presenta resultados de investigación sobre procesos de construcción de liderazgos comunitarios con dos estudios de caso de organizaciones de mujeres indígenas. El propósito fue conocer estrategias comunitarias que desarrollan las actoras sociales locales al interior de sus organizaciones, su vinculación con la comunidad y con otros actores para la formación y consolidación de liderazgos comunitarios. Se utilizó una metodología cualitativa, con entrevistas grupales y observaciones directas. $\mathrm{Se}$ resalta la importancia del trabajo comunitario enfocado a potenciar esfuerzos, capacidades y habilidades de las asociaciones civiles bajo estudio para mejorar la calidad de vida de ellas y de la comunidad.
\end{abstract}

Palabras claves: Mujeres y liderazgo; organizaciones de mujeres indígenas; estrategias organizativas; liderazgo comunitario; empoderamiento de la mujer indígena

\footnotetext{
1 Doctora en Ciencias Sociales. Profesor-Investigadora de la Facultad de Ciencias Administrativas y Sociales de la Universidad Autónoma de Baja California. http://orcid.org/o0o0-0001-81836405.1camarena@uabc.edu.mx.

2 Maestra en Antropología Cultural. Profesor-Investigadora de la Facultad de Ciencias Administrativas y Sociales de la Universidad Autónoma de Baja California. http://orcid.org/0000-0002-7528-5341.conmar@uabc.edu.mx.

3 Doctora en Medio Ambiente y Desarrollo. Profesor-Investigadora de la Facultad de Ciencias de la Universidad Autónoma de Baja California. http://orcid.org/0000-0002-0997-6902. evarista.arellano@uabc.edu.mx

4 Doctora en Ciencias Sociales. Profesora-Investigadora. Departamento de Estudios de Población, Línea de Salud Pública, El Colegio de la Frontera Norte. http://orcid.org/0000-0002-43054530. cglascoe@colef.mx
} 


\begin{abstract}
This paper presents research results dealing with processes of community leadership building based on two case studies of indigenous female organizations. The purpose was to explore the communal strategies developed by these women within their organizations and their links with the community and other actors as part of the process of forming and consolidating community leadership. A qualitative methodology was utilized which included group interviews and direct observation. The importance of community work aimed at strengthening the abilities and skills for improving the quality of life of these women and of the community itself is highlighted.
\end{abstract}

Keywords: Women and leadership; indigenous female organizations; organizational strategies; community leadership; empowerment of indigenous women

\title{
Introducción
}

El Valle de San Quintín se encuentra a 300 kilómetros de la frontera Méxicoestadounidense sobre la costa del Pacífico. La región de San Quintín se ubica en el municipio de Ensenada, en la zona sur del estado, a 190 kilómetros de la cabecera municipal, siendo su área de influencia desde el poblado de San Vicente, hasta el de Villa Jesús María y comprende, además, los poblados de Colonet, Camalú, El Rosario, Bahía de los Ángeles y Punta Prieta, entre otros. La región de San Quintín abarca desde el poblado de San Vicente hasta el paralelo 28. Demográficamente ha sido una región con dinámicas migratorias en donde se recibe una gran cantidad de hombres, mujeres y sus familias provenientes de otros estados del país que visualizan a la región como una oportunidad para emplearse en las labores agrícolas. La importancia que ha adquirido la migración indígena hacia el estado de Baja California, está asociada a la producción hortícola de exportación específicamente a los Estados Unidos, en la cual la participación de mano de obra indígena y en especial la femenina han sido importante.

En México según la Encuesta Intercensal 2015 del Instituto Nacional de Estadística y Geografía (INEGI) 25.7 millones de personas se autorreconocen como indígenas, representando el $21.5 \%$ de la población total mexicana, de este porcentaje 13.2 millones son mujeres (INEGI, 2016). 
El Valle de San Quintín cuenta con 87,616 habitantes según el censo de 2010, lo cual representa el $18.8 \%$ del total municipal. De estos, el $50.8 \%$ son hombres y el $49.2 \%$ son mujeres. En cuanto a la migración, desde la región se observa que $52.1 \%$ son personas originarias de otros estados, el $45.6 \%$ son nacidos en la entidad y el restante $2.3 \%$ corresponde a personas originarias de otro país y/o no especificado. En el Valle de San Quintín en 2010 el total de personas de 5 años y más de edad que hablan alguna lengua indígena es 12,773 y el $5.3 \%$ de ellos no habla español (COPLADE, 2017).

En este trabajo se presentan resultados de investigación sobre procesos de construcción de liderazgos comunitarios con base en dos estudios de caso de organizaciones de mujeres indígenas en el Valle de San Quintín, Baja California, Mujeres en Defensa de la Mujer A.C. y Mujeres Tejiendo Nuevo Amanecer A.C. El propósito de este estudio fue conocer las estrategias que estas dos asociaciones desarrollan para ejercer liderazgo con actores sociales locales, nacionales e internacionales.

El liderazgo en las organizaciones de la sociedad civil en comunidades rurales y localidades con población indígena, ha recaído más en los hombres que en las mujeres. A pesar de la presencia de las mujeres indígenas en procesos organizativos comunitarios, su trabajo no suele ser reconocido. La visibilización del quehacer de las mujeres indígenas como agentes de cambio en sus localidades ha crecido poco a poco a través de la defensa de sus derechos, exigiendo oportunidades, expresando sus necesidades, reivindicando sus derechos laborales, sexuales y reproductivos. De esta manera han ido adquiriendo nuevos roles en la sociedad.

El liderazgo se ha estudiado desde diversas disciplinas y enfoques teóricos tanto por psicólogos sociales, teóricos de la gestión, como por economistas y analistas de políticas públicas. Desde una perspectiva conductual, se clasifican diversos tipos de liderazgo como el autocrático, el democrático, el auténtico, entre otros, para explicar cómo opera en vez de cómo se origina el liderazgo (Avolio y Gardner, 2005; Beer, 2014). Otra forma de estudiarlo es analizando el tipo de interacciones que se dan entre los individuos, estableciendo diferencias entre líderes socioemocionales preocupados por los sentimientos de las personas en contraste con los líderes orientados hacia las metas o tareas del conjunto (Stimson, R. J., Stough, R., y Salazar, 2009). 


\section{DOXA}

pISSN: 2395-8758

eISSN: en trámite

En años recientes las teorías sobre el liderazgo han experimentado una transformación al transitar de perspectivas tradicionales del liderazgo donde éste se centra en los atributos individuales, pasando por la relación recíproca entre individuos hasta la concepción emergente que lo considera como un proceso de construcción colaborativo entre personas que buscan un cambio intencional (Komives, 1994; Rost, 1991).

Autores como Shamir y Eilam (2005) describen los liderazgos a partir de las historias de vida de los líderes donde éstas reflejan su grado de autoconocimiento y claridad de su propio rol de líder. Además comentan los autores que el liderazgo se suele asociar con una posición de autoridad formal, o con una serie de rasgos personales. Komives y Dugan (2010) consideran al liderazgo como un proceso recíproco y dinámico entre personas que persiguen metas comunes para el logro del bien común a través de la acción colectiva. Para autores como Beer y Sotarauta, es importante estudiar el contexto y los procesos en los cuales los liderazgos se desarrollan, resaltando la importancia de enfocarse en las relaciones que conectan a los actores en lugares específicos que pueden ser ciudades, regiones o comunidades rurales (Beer, 2014; Sotarauta, Beer, y Gibney, 2017)

En este sentido, los líderes comunitarios realizan labores de intermediación, gestión y atención de diversas necesidades presentes en los diversos grupos de población y promueven la formación de capital social y la participación ciudadana que posibilitan la interlocución entre los actores comunitarios y otros sectores. Para el estudio de liderazgos comunitarios en contextos rurales, es necesario analizar las dinámicas sociales dentro de las mismas comunidades (Herbert-Cheshire, 2003, Citado en Beer, 2014) como la experiencia migratoria.

Una de las dinámicas sociales en la que es posible explorar el desarrollo de liderazgos comunitarios es la experiencia migratoria de población rural e indígena que los posiciona en una situación de marginación en la cual se tiene nula participación social. Aunque los procesos migratorios tradicionalmente habían involucrado principalmente a hombres, cada vez más son las mujeres quienes se integran al proceso migratorio (Kellogg, 2005; Lara-Flores, 2009). El tipo de trabajo que está disponible para estas mujeres indígenas son trabajos poco calificados, donde las condiciones y exigencias laborales son extenuantes y con bajos salarios (Lara Flores, 2003; Mines, 
pISSN: 2395-8758

eISSN: en trámite

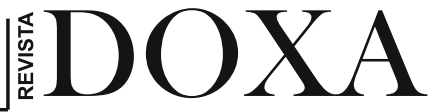

Nichols, y Runsten, 2010). Al encontrarse en el lugar destino, por su condición étnica, las mujeres sufren discriminación por parte de la población mestiza, lo que afecta sus condiciones de empleo y de vida. Esta situación provoca que busquen estrategias y alternativas de movilización colectiva que transforme su condiciones inequitativas. Esta movilización ha llevado a la conformación de colectivos y de asociaciones civiles de mujeres indígenas.

Maier (2006) establece que en el caso de las mujeres indígenas migrantes en Baja California este tránsito desde sus lugares de origen ha significado una reedición de su propia identidad femenina y que las condiciones económicas en el lugar de recepción conducen a una movilidad cultural, emocional y social que les permite constituirse como sujetos de su propia vida, lo que deriva en un convencimiento del derecho a tener derechos. La experiencia migratoria para estas mujeres ha significado un cambio en la relación con sus parejas y con sus hijos. El trabajo remunerado las habilita a tener control sobre el ingreso familiar, lo cual les permite tener cierto tipo de independencia frente a su pareja. Asimismo, la incorporación al mercado laboral las ha relacionado con colectivos de mujeres. Esto les ha posibilitado organizarse e incursionar en el espacio público para exigir cambios y realizar actividades comunitarias para el bien común. Este proceso de incursionar en el ámbito público de las mujeres indígenas representa un reto "pues constituye una transgresión al orden simbólico y a la organización social de los géneros; a los conceptos de masculinidad y feminidad y a los espacios que culturalmente se asignan a varones y mujeres" (Espinosa, 2009). Cuando se trata de la construcción de liderazgos indígenas femeninos, esta misma autora observa que en el desarrollo organizativo las mujeres enfrentan violencia y rechazo social.

En este trabajo se emplea el concepto de liderazgo comunitario como lo describe Martiskainen (2017), señalando que éste suele ser menos jerárquico, basado en participación voluntaria, de carácter informal, de libre elección y de participación grupal o individual. Este tipo de liderazgo conlleva a la formación de capital social al interior de una comunidad. La noción de comunidad en este sentido no se define en términos espaciales, sino trasciende fronteras culturales y se construye con base en experiencias, historias y experiencias compartidas (Dahlander y Frederiksen, 2012; McMillan y Chavis, 1986; Walker, 2008). 


\section{DOXA}

pISSN: 2395-8758

eISSN: en trámite

De esta manera, el liderazgo comunitario promueve la formación de capital social y la participación social, teniendo un impacto positivo en las comunidades donde se desarrolla. Un liderazgo comunitario efectivo fomenta el empoderamiento de los lazos afectivos de la comunidad, construye una memoria histórica y colectiva compartida, fomenta la equidad e igualdad de género, permite la transformación de conflictos, facilita la organización comunitaria con decisiones colegiadas y genera capital social comunitario.

\section{Metodología}

Se utilizó una metodología cualitativa donde se aplicaron entrevistas semiestructuradas a integrantes de dos asociaciones civiles situadas en dos comunidades en el Valle de San Quintín, el ejido Emiliano Zapata y la Delegación Lázaro Cárdenas. El trabajo de campo se llevó a cabo entre marzo y abril del 2016, en el primer mes se realizó una entrevista grupal a la Asociación Mujeres en Defensa de la Mujer y en el mes siguiente la entrevista grupal se aplicó a la Asociación Mujeres Tejiendo un nuevo Amanecer. A través de estas entrevistas se buscó indagar sobre las actividades que estas asociaciones civiles realizan en sus localidades. Las entrevistas fueron grabadas, posteriormente transcritas y analizadas para después realizar su análisis de contenido.

La guía de la entrevista se estructuró en torno a ocho categorías operativas:

1) Capital social, considerado como aquellas instituciones, relaciones y normas que moldean la cantidad y calidad de las interacciones sociales.

2) Confianza y colaboración al interior de la organización la cual es definida por Fukuyama (1995) como el comportamiento esperado al interior de una colectividad donde las acciones sean ordenadas y de cooperación, basándose en normas compartidas por todos los integrantes.

3) Estrategias que desarrollan considerando la movilización de recursos formales, informales, materiales e inmateriales; las formas de comunicación que utilizan; los diversos niveles de relaciones que construyen para incidir en el ámbito público-gubernamental.

4) Articulación estado-comunidad, dada por las acciones que 
pISSN: $2395-8758$

eISSN: en trámite



fortalecen las capacidades y oportunidades de actores tradicionalmente excluidos y por los procesos de formación de políticas públicas incluyentes, (Mokate y Saavedra, 2005), considerando que la articulación de todos los actores debe dirigirse a la construcción del desarrollo social, del bien común.

5) Vinculación con redes nacionales e internacionales. Entre los actores que integran las redes se considera necesario incluir organizaciones nacionales e internacionales gubernamentales; organizaciones nacionales e internacionales no gubernamentales; movimientos sociales locales; instituciones académicas; fundaciones y otros benefactores; medios de comunicación.

6) Logros y resultados. Vistos como expresiones tangibles e intangibles de las estrategias de los actores político-comunitarios tanto aquellas relacionadas con el accionar al interior de la organización como las que inciden en el ámbito de lo públicogubernamental

7) Legitimidad entendida como el reconocimiento de la comunidad, instituciones y autoridades al quehacer de la organización.

8) Permanencia, referida a la trayectoria lineal del tiempo en el que el actor político-comunitario se desarrolla y las estrategias que ejecuta para incidir en lo público-gubernamental y en lo políticocomunitario.

\section{Resultados}

Las mujeres integrantes de las dos asociaciones iniciaron su participación comunitaria en la edad adulta, por medio de talleres y capacitaciones; posteriormente a partir de la interacción con otras mujeres, fueron conformando asociaciones las cuales fueron constituidas legalmente.

La información surgida a través de las entrevistas grupales corrobora lo señalado en la introducción de este artículo, donde se refiere que el liderazgo que ejercen las mujeres de estas dos asociaciones es horizontal y consensual, el cual surge como un proceso de construcción colaborativo. Por lo tanto, el liderazgo que se observó en estos grupos no está representado en una sola persona. 
Caso Mujeres en Defensa de la Mujer A.C.

La construcción de liderazgo en este grupo de mujeres se dio a través de un proceso inicial reflexivo de seis mujeres indígenas trabajando en distintos proyectos productivos sobre el rescate del bordado y la medicina tradicional.

Todas ellas trabajadoras jornaleras experimentaron la violación de sus derechos laborales y discriminación por ser indígenas y migrantes. Dadas estas condiciones sociales es que se organizan formalmente y en el 2004 se constituyen como asociación civil, realizando actividades particularmente relacionadas con la defensa de los derechos laborales, violencia intrafamiliar, salud sexual y reproductiva y el rescate de la medicina tradicional.

En cuanto a la categoría de capital social, se encontró que buscaron asociaciones civiles a nivel nacional que les brindaran capacitación, en particular con "Fondo Semillas. A.C." de la ciudad de México, la cual es una organización feminista que mejora la vida de las mujeres. También establecieron vínculos con académicos de la universidad estatal.

Con respecto a la articulación estado-comunidad, señalaron su incorporación como asociación al programa "Casa de la Mujer Indígena" (CAMI), generado por la Comisión Nacional para el Desarrollo de Pueblos Indígenas (CDI). Eso les permitió tener un espacio de representación ante la comunidad y brindar servicios de asesoría psicológica y jurídica. A través de la CAMI han logrado tener mayor vinculación con instituciones de procuración de justicia y del sector salud. En estas actividades interactúan con el Ministerio Público, con la Comisión Estatal de Derechos Humanos, Defensoría Pública, el Juzgado Civil y Penal, y con la Secretaría de Salud, así como con el Instituto Mexicano de Seguro Social e Instituto Nacional de la Mujer en Ensenada.

Referente a las estrategias de comunicación, ellas comentaron que se apoyan en la Radio Comunitaria XE-QUIN, transmitiendo un programa denominado "Mujeres Tejiendo Caminos", en el cual se difunde información sobre sus derechos, además de informar a la comunidad sobre las actividades que realiza la organización. También elaboran diferentes tipos de folletería donde dan a conocer los servicios que ofrecen. También comentaron que han buscado desarrollar estrategias creativas hacia la comunidad, como la utilización de títeres para trabajar con niños y adolescentes; realizar actividades en mixteco; "dramatizaciones" informativas sobre los servicios 
pISSN: $2395-8758$

eISSN: en trámite

que ofrecen a la comunidad.

“...no solo se trata de que vamos a ir y vamos a estar una hora y vamos a dar el taller, sino también pues ponerles proyecciones comunitarias también para que de esa forma capten mayor atención de las compañeras que asistan y, ya después de ahí, empezar a compartirles como la retroalimentación de nuestra parte y también de las mismas compañeras. En ese sentido de la difusión de esas temáticas, estamos tratando de darle un cambio también a esto."

Sobre la vinculación con redes nacionales e internacionales, mencionaron que a través de CAMI han establecido vínculos con la Coordinadora Nacional de Mujeres Indígenas para analizar las políticas públicas en los estados, asimismo han sido invitadas a participar en foros de mujeres indígenas a nivel nacional e internacional.

“...hemos tejido como redes con asociaciones, este pues nos hemos acercado a las asociaciones o grupos sobre todo que estén conformados por mujeres que están en la misma lucha, en el mismo proceso que nosotras, pero si tenemos todavía mayor vinculación con las instituciones de procuración de justicia y sector salud"

Respecto a la confianza y colaboración al interior de la organización, señalaron que su forma de trabajo se orienta hacia la colaboración entre todas al igual, el reparto de actividades se distribuye de manera equitativa y la toma de decisiones es consensuada. Además realizan una reunión anual para evaluar el trabajo realizado y planear futuras actividades. En cuanto a la toma de decisiones al interior de la organización, comentaron que todas participan y resuelven conflictos según el modelo de consenso.

“... en caso del proyecto de casa de la mujer estamos cinco de las socias, en el proyecto del temazcal están cinco también de las socias y en lo que es la defensa de los derechos laborales pues ahí estamos todas."

Con relación a logros, resaltan que han podido desarrollar y consolidar proyectos como la capacitación y asesoría sobre los derechos laborales, los derechos sexuales y reproductivos, la violencia intrafamiliar y el recate de la medicina tradicional. Consideran que sus proyectos les han permitido incrementar el acercamiento con las instituciones, actividad clave para acciones de gestión. Relacionado a los logros obtenidos ellas creen que la legitimidad de su asociación se ha logrado al obtener el reconocimiento de 


\section{DOXA}

pISSN: $2395-8758$

eISSN: en trámite

la comunidad y de las instituciones.

"[En la radio] por los mensajes que llegan y eso, nos damos cuenta en qué momento la gente está escuchando, por ejemplo ahorita ya tenemos el face de la radio y ahí también nos llega la información así al instante ¿no?, entonces así es como nos damos cuenta de la población que nos escucha, pero mayoritariamente es la gente indígena"

Concerniente a su permanencia como asociación, comentaron que piensan fortalecer los vínculos con las autoridades comunitarias para extender sus talleres a otras localidades y continuar con la formación de promotoras comunitarias. Piensan que sus retos como organización son obtener mayor reconocimiento por parte de otras asociaciones civiles y de las instancias gubernamentales. A nivel operativo, el obtener fondos propios para operar los programas así como atraer la participación de los hombres en los talleres que se imparten para disminuir la violencia contra la mujer.

\section{Caso Mujeres Tejiendo un Nuevo Amanecer, A.C.}

Al igual que en el caso anterior, la construcción de liderazgo en este grupo de mujeres se dio a través de un proceso inicial de trabajo comunitario enfocado a atender las necesidades del adulto mayor en su comunidad, con el propósito de proporcionar alimentos a personas de la tercera edad. También las integrantes de esta asociación son jornaleras que experimentan la violación de sus derechos laborales y discriminación por ser indígenas y migrantes. En el 2011, se constituyeron legalmente como asociación civil.

Referente al capital social, señalaron que este lo han ido construyendo a través de sus servicios a la localidad como promotoras comunitarias. Comentan que algunas de ellas tienen experiencia como promotoras de salud en la comunidad, lo cual les ha permitido conocer y tener contacto con otras mujeres en su comunidad. Opinan que a través del contacto continuo con la comunidad, fue lo que les permitió la construcción del comedor ya que el terreno lo proporcionó la comunidad y así mismo las apoyaron con la mano de obra.

En cuanto la confianza y la colaboración al interior de la organización, señalaron que si bien, una de ellas asume un papel más activo, entre todas toman decisiones y definen su plan de trabajo. Asimismo comentan que las actividades las reparten con base en las habilidades de cada 
pISSN: 2395-8758

eISSN: en trámite

una, pero también de acuerdo al tiempo que tienen disponible:

"A las que trabajan, se les da su tarea y ya que la hagan en la tarde, otras hacemos lo que se puede hacer en el día, pero a veces en la tarde ahí estamos también...siempre venimos aunque no nos toque ese día, siempre venimos, siempre estamos ahí con las otras que les toca hacer la tarea".

Relacionado a las estrategias que desarrollan para lograr su objetivo de construir y sostener el comedor para adultos mayores, ellas comentan que es necesario realizar actividades de venta de comida para comprar material para la construcción, solicitar donaciones de personas de la comunidad o del cabildo municipal e invitar a los hombres de la comunidad para apoyarlas en los trabajos de albañilería. Identificaron como estrategia relacionarse con la Universidad Autónoma de Baja California (UABC) para conseguir asesoría para el desarrollo de proyectos y talleres de corte ambiental, tales como la construcción de un invernadero para cultivar plantas medicinales, talleres para la elaboración de pomadas, jabones, velas y esencias, así como talleres de reciclaje. Además señalan que otra estrategia que utilizaron fue buscar el apoyo de una organización canadiense filantrópica de corte religioso “Corazón, Fuerza y Amor”, ubicada en la localidad para ampliar el comedor para los adultos mayores.

Consideran que la vinculación con otras organizaciones comunitarias, con instancias gubernamentales o con empresas, es bastante débil. Señalan que en sus inicios fue de gran apoyo la asociación "Mujeres en defensa de la Mujer". Referente a la vinculación con otras instancias como la Secretaría de Desarrollo Social, así como la Secretaría de Hacienda y Crédito Público, ellas identifican la necesidad de contactarse y registrarse ante estas dependencias, ya sea para acceder a algún proyecto o para recibir donativos de empresas.

En cuanto a los logros que han tenido como asociación, consideran que los dos principales han sido la construcción del comedor para adultos mayores de la comunidad y del invernadero de medicina tradicional. Referente a la legitimización señalan que los miembros de la comunidad reconocen el trabajo que ellas realizan:

[Miembros de la comunidad les han expresado] “...ustedes están haciendo muy bien porque lo están haciendo de corazón, no lo están haciendo porque quieren algo." "Es un logro que muchas mujeres nos tomen como ejemplo, y hay 
muchas, muchas que ya han cambiado su manera de pensar o de vivir en sus casas".

Relativo a la permanencia, ellas comentan que necesitan capacitarse para la obtención de recursos para poder continuar con sus proyectos, así como reclutar a más mujeres para continuar y consolidar la asociación. Señalan que su visión a futuro es ampliar sus proyectos.

“...la visión que tenemos de trabajarlo aunque ahorita como no tenemos un espacio propio, lo vemos un poco difícil y no lo hemos aterrizado, no lo hemos empezado pero si lo tenemos contemplado, lo que es tocar lo que es los derechos de la mujer, lo que es la violencia intrafamiliar o de los jóvenes"

\section{Discusión y Conclusiones}

El proceso de construcción de liderazgos comunitarios de las mujeres indígenas en el Valle de San Quintín en Baja California, se puede explicar a partir de varios elementos entre los que se distinguen, 1) la experiencia migratoria (Coubès, 2009), que conduce a los cambios en la ecuación de equidad de género como resultado de la feminización del trabajo agrícola (OIM, 2013) y 2) la resignificación de su papel como agentes de cambio en sus propias localidades, 3) la capacitación proporcionada por algunas organizaciones de la sociedad civil e instancias gubernamentales, que han sido caldo de cultivo para el surgimiento de estas organizaciones, 4) la participación en el trabajo comunitario que ha servido como plataforma para el proceso de conformación de liderazgos comunitarios de estas mujeres.

A más de veinte años de la intensificación del proceso migratorio desde los estados más pobres de México hacia Baja California, las mujeres indígenas que se trasladaron ya sea solas, con sus respectivas familias nucleares o con sus parejas, han experimentado un asentamiento gradual en la región. El haber migrado y haberse incorporado al trabajo productivo en los campos agrícolas propició, entre otras cosas, el rechazo al control patriarcal. Se ha observado que estas mujeres han experimentado un cambio gradual "de su propia identidad femenina y que las condiciones económicas en el lugar de recepción conducen a una movilidad cultural, emocional y social que les permite constituirse como sujetos de su propia vida, lo que deriva en un convencimiento del derecho a tener derechos" (Maier, 2006). Los cambios identitarios como resultado de la evolución del proceso 
pISSN: 2395-8758

eISSN: en trámite

migratorio y la feminización del trabajo agrícola, posibilitaron cambios en los roles de género en las mujeres indígenas jornaleras por su papel como proveedoras o jefas de familia (Maier, 2006) y las sitúa como actoras y decisoras con más visibilidad al interior de la familia y adicionalmente en el entorno de la propia comunidad en sus recientes asentamientos en San Quintín.

La resignificación de su identidad como mujer y como gestora de cambios tanto en la esfera privada como en la pública, ha permitido el organizarse para exigir el respeto a sus derechos laborales, sexuales y reproductivos. Estas formas de organización han conducido a algunas de estas mujeres a formarse como líderes reconocidas en la comunidad y por organizaciones e instituciones nacionales e internacionales. Se resalta la importancia del trabajo comunitario enfocado a potenciar los esfuerzos, capacidades y habilidades del grupo para mejorar la calidad de vida de ellas y de la comunidad.

Los liderazgos son producto de los aprendizajes donde ellas se construyen y se reinventan a partir de su interacción con redes de organizaciones sociales, el intercambio de información y experiencias. Es en estos procesos que se posibilita el desarrollo de una visión de ejercicio participativo y de agencia política. El estar inmersas en proyectos colectivos dentro de sus organizaciones les permite, por un lado, capacitarse ellas mismas y a la vez ser multiplicadoras de las herramientas técnicas y metodológicas para asesorar y dar apoyo a otras mujeres, a la vez ser puente con las instancias gubernamentales. Por otro lado, las organizaciones constituidas por ellas permite crear espacios de participación entre la comunidad y las instituciones gubernamentales.

$\mathrm{Si}$ bien es cierto, que ya existen organizaciones de mujeres indígenas, aún falta recorrer formas efectivas y equitativas de interlocución con instancias gubernamentales y académicas. Para ser escuchadas, las mujeres indígenas han tenido que aprender el discurso hegemónico y ello ha representado un reto intercultural, que aún no ha sido rebasado. A su vez el lado hegemónico tendría que asumir la existencia de la interculturalidad para reconocer el diálogo de doble vía. La formación de liderazgos comunitarios enfrenta asimismo obstáculos para la emancipación de las mujeres indígenas, tal como la formación de liderazgos funcionales ejemplificado en la figura de 


\section{DOXA}

pISSN: 2395-8758

eISSN: en trámite

la promotora comunitaria contratada por instancias gubernamentales. Este obstáculo, sin embargo puede ser visto como una ventana de oportunidad que conduce a la formación de capital social el cual puedes ser apropiado y redirigido a la transformación de las problemáticas y necesidades comunitarias.

\section{Referencias}

Avolio, B. J., y Gardner, W. L. (2005). Authentic leadership development: Getting to the root of positive forms of leadership. Leadership Quarterly, $16(3)$ 315-338. https://doi.org/10.1016/j.leaqua.2005.03.001

Beer, A. (2014). Leadership and the governance of rural communities. Journal of Rural Studies, 34, 254-262. https://doi.org/10.1016/j.jrurstud.2014.01.007

COPLADE. (2017). Programa para la atención de la región de San Quintín (2015-2019). Recuperado de: http://www.copladebc.gob.mx/programas/Programa para la Atencion de la Region de San Quintin 2015-2019.pdf Coubès, M.L. (2009). Agriculture pour l'exportation et peuplement rural dans la région frontalière Mexique/États-Unis : San Quintin en Basse Californie. Cahiers Des Amériques Latines. https://doi.org/10.4000/cal.1854

Dahlander, L., y Frederiksen, L. (2012). The Core and Cosmopolitans : A Relational View of Innovation in User Communities The Core and Cosmopolitans : A Relational View of Innovation in User Communities. Organization Science, 23(4), 988-1007. https://doi.org/10.2307/23252445

Espinosa, G. (2009). Liderazgo y violencia de género en el guerrero indígena. Revista Venezolana de Estudios de La Mujer, 14(32), 211-224. Recuperado de:http://www.scielo.org.ve/scielo.php?pid=S1316$37012009000100015 y$ script $=$ sci_arttext

Fukuyama, F. (1995). Social Capital and the Global Economy. Foreign Affairs.

https://doi.org/10.2307/20047302

INEGI. (2016). Estadísticas a propósito del día internacional de los pueblos 
pISSN: 2395-8758

eISSN: en trámite

indígenas (9 de agosto). Datos Nacionales.

Kellogg, S. (2005). Weaving the Past. A History of Latin America's Indigenous Women from the Prehispanic Period to the Present. New York: Oxford University Press.

Komives, S. R. (1994). Women student leaders: Self-perceptions of empowering leadership and achieving style. NASPA Journal.

Komives, S. R., y Dugan, J. P. (2010). Contemporary leadership theories. In Political and Civic Leadership: A Reference Handbook. https://doi.org/10.4135/9781412979337.n14

Lara Flores, S. M. (2009). El papel de las mujeres en las migraciones y en la movilidad de los grupos indígenas de México. In I. Instituto Interamericano de Derechos Humanos (Ed.), Las mujeres indígenas de América Latina en los procesos migratorios (pp. 21-40). San José de Costa Rica: Editorama, S.A. Recuperado de:https://www.iidh.ed.cr/IIDH/media/1500/mujeres-indigenasen-procesos-migratorios-2009.pdf

Lara Flores, S. M. (2003). Violencia y contrapoder: una ventana al mundo de las mujeres indígenas migrantes, en México. Revista Estudios Feministas, 11(2), 381-397. https://doi.org/10.1590/So104026X2003000200003

Maier, E. (2006). Tránsitos territoriales e identidad de las mujeres indígenas migrantes. Papeles de Población, 12(47), 203-225. Recuperado de: http://www.redalyc.org/pdf/ 112/11204708.pdf

Martiskainen, M. (2017). The role of community leadership in the development of grassroots innovations. Environmental Innovation and Societal Transitions, 22(1), 78-89. https://doi.org/10.1016/j.eist.2016.05.002

McMillan, D. W., y Chavis, D. M. (1986). Sense of community: A definition and theory. Special Issue: Psychological sense of community, I: Theory and concepts. Journal of Community Psychology, 14(1), 6-23. https://doi.org/10.1002/1520-6629(198601)14:1<6::AIDJCOP2290140103>3.0.CO;2-I

Mines, B. R., Nichols, S., y Runsten, D. (2010). California's Indigenous Farmworkers. Final Report of the Indigenous Farmworker Study (IFS) To the California Endowment. Recuperado de : 


\section{DOXA}

pISSN: 2395-8758

eISSN: en trámite

http://www.crla.org/sites/all/files/content/uploads/pdfBrochure s/IFS_Mines_Final_2010.pdf

Mokate, K., y Saavedra, J. J. (2005). Gerencia social: un enfoque integral para la gestión de políticas y programas sociales. Instituto Interamericano Para El Desarrollo Social. Washington, D.C. Barcelona. Recuperado de: http://vipp.es/biblioteca/EMPB_0027.pdf

OIM. (2013). Migración y Ciudadanía.

Rost, J. (1991). Leadership for the twenty-first century. The Leadership Quarterly. https://doi.org/10.1016/1048-9843(91)90021-S

Shamir, B., y Eilam, G. (2005). "What's your story?” A life-stories approach to authentic leadership development. Leadership Quarterly. https://doi.org/10.1016/j.leaqua.2005.03.005

Sotarauta, M., Beer, A., y Gibney, J. (2017). Making sense of leadership in urban and regional development. Regional Studies, 51(2), 187-193. https://doi.org/10.1080/00343404.2016.1267340

Stimson, R. J., Stough, R., y Salazar, M. (2009). (2009). Leadership and institutions in regional endogenous development. Edward Elgar Publishing.

Walker, G. (2008). What are the barriers and incentives for communityowned means of energy production and use? Energy Policy, 36(12), 4401-4405. https://doi.org/10.1016/j.enpol.2008.09.032 